\title{
Analysis of Terahertz Wave on Increasing Radar Cross Section of 3D Conductive Model
}

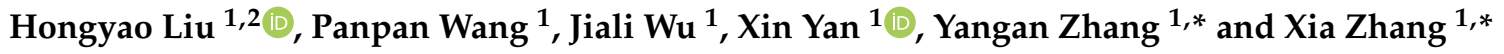 \\ 1 State Key Laboratory of Information Photonics and Optical Communications, Beijing University of \\ Posts and Telecommunications, Beijing 100876, China; liuhongyao20@163.com (H.L.); \\ panpanono@163.com (P.W.); Flowing_Kinga@bupt.edu.cn (J.W.); xyan@bupt.edu.cn (X.Y.) \\ 2 School of Information and Communication Engineering, Beijing University of Posts and Telecommunications, \\ Beijing 100876, China \\ * Correspondence: zhang@bupt.edu.cn (Y.Z.); xzhang@bupt.edu.cn (X.Z.)
}

Citation: Liu, H.; Wang, P.; Wu, J.;

Yan, X.; Zhang, Y.; Zhang, X. Analysis of Terahertz Wave on Increasing Radar Cross Section of 3D Conductive Model. Electronics 2021, 10, 74. https://doi.org/10.3390/ electronics10010074

Received: 12 November 2020 Accepted: 22 December 2020 Published: 3 January 2021

Publisher's Note: MDPI stays neutral with regard to jurisdictional clai$\mathrm{ms}$ in published maps and institutional affiliations.

Copyright: (C) 2021 by the authors. Licensee MDPI, Basel, Switzerland. This article is an open access article distributed under the terms and conditions of the Creative Commons Attribution (CC BY) license (https:// creativecommons.org/licenses/by/ $4.0 /)$.

\begin{abstract}
Enhancing the frequency band of the electromagnetic wave is regarded as an efficient way to solve the communication blackout problem. In this paper, frequency of incident wave is raised to Terahertz (THz) band and the radar cross section (RCS) of the three-dimensional conductive model is calculated and simulated based on the Runge-Kutta Exponential Time Differencing-Finite Difference Time Domain method (RKETD-FDTD). Interaction of $\mathrm{THz}$ wave and magnetized plasma sheath is discussed. Attenuations in incident wave frequencies of $0.34 \mathrm{THz}$ and $3 \mathrm{GHz}$ and different plasma densities are analyzed. The monostatic RCS is used to compare the penetration in different incident wave frequencies while the bistatic RCS is fixed on $0.34 \mathrm{THz}$ to study its characteristics. The simulation result has almost the same RCS as that of the model without coating plasma when the frequency of incident wave reaches $0.34 \mathrm{THz}$. The advantages of $\mathrm{THz}$ wave at $0.34 \mathrm{THz}$ on increasing RCS and reducing the attenuation are demonstrated from different aspects including polarizations, incident angles, magnetization and anisotropy of plasma, thickness of plasma, scan planes and inhomogeneous distribution of plasma. It can be concluded that $0.34 \mathrm{THz}$ has unique advantages in increasing the radar cross section and can be applied to solve the problem of communication interruption.
\end{abstract}

Keywords: THz wave; RCS; RKETD-FDTD; magnetized plasma; blackout problem

\section{Introduction}

The communication blackout problem remains to be completely solved. When the supersonic vehicle is moving in the near space, it is surrounded by a mixture of gas and plasma called a "plasma sheath" [1]. Most of the time, the communication frequency is lower than the cut-off frequency of the sheath and the communication is interdicted. Researchers all over the world have come up with methods in response to the blackout problem. Changing the aerodynamic profile of the vehicle [2], using the crossed electromagnetic field to reduce the density of the plasma sheath [3], using the magnetic field to form a "magnetic window" to let the wave pass through [4-6], placing the antenna on the low-density Leeward side of the plasma sheath [7] and increasing the frequency of communication are methods that have been developed to solve the blackout problem. Researches have pointed out that one of the important reasons for communication failure is that the electromagnetic wave (EM wave) frequency is lower than the plasma frequency, which leads to a huge attenuation while the EM wave is spreading in the plasma [8]. The peak of the plasma density can reach up to $10^{21} \mathrm{~m}^{-3}$ as is recorded in flight data [9]. Comprehensively considering the above reason, it turns out that $\mathrm{THz}$ wave is an efficient method to solve the problem. Additionally, a number of developments for the $0.34 \mathrm{THz}$ transceiver front end have been proposed which makes the telecommunications in this frequency band realizable [10,11]. Therefore, it is of great significance to study the propagation and scattering characteristics of $\mathrm{THz}$ wave at $0.34 \mathrm{THz}$ from a comprehensive perspective. Hence, in this work, 
bistatic RCS of the 3D model is fixed on $0.34 \mathrm{THz}$ to study the scattering characteristics in the magnetized plasma and verify the function in solving the blackout problem.

To date, several groups have used a numerical algorithm to study EM wave and plasma. Wang et al. studied the $\mathrm{THz}$ propagation characteristics of dusty plasma slab [12]. Yuan et al. researched $\mathrm{THz}$ wave propagation in high-temperature plasma [13]. Kang et al. used the precise integration time-domain (PITD) method instead of the Finite-Difference TimeDomain (FDTD) method to study the propagation in magnetized plasma [14] On the other hand, the RCS is a physical quantity to characterize the echo intensity of the target irradiated by the radar wave and is of great importance in studying scattering characteristics [15,16]. Theoretical studies on RCS and scattering characteristics in a certain frequency band have been reported [17-22]. However, most of the simulation models are limited to one-dimension and the frequency of incident wave is lower than $0.1 \mathrm{THz}$.

In this paper, we increase the frequency of the differential Gaussian pulse to the $\mathrm{THz}$ band and calculate both monostatic RCS and bistatic RCS of the three-dimension conductive model coated with magnetized plasma using the RKETD-FDTD method. Besides, the attenuation in different electron densities is analyzed using the Wentzel-Kramer-Brillouin (WKB) method. Generally speaking, the RKETD-FDTD method is used to solve the electromagnetic characteristics of the dispersion medium as it has higher calculation efficiency and accuracy than the convolution FDTD algorithm formula [23,24]. In order to demonstrate the effect of increasing the frequency of incident wave on reducing the attenuation effect of the plasma, the monostatic RCS curves are calculated in the frequency band $0-0.6 \mathrm{THz}$. From simulation results of monostatic RCS, it can be found that EM wave frequency at $0.34 \mathrm{THz}$ has almost the same RCS value as that of the no plasma coating curve. To prove all-around excellent performances concerning $0.34 \mathrm{THz}$ waves, the bistatic RCS at $0.34 \mathrm{THz}$ with different polarization ways, distributions of plasma, plasma parameters, cyclotron resonance directions, incident angles in a certain range and planes are also discussed. The results demonstrate the superiority of the $0.34 \mathrm{THz}$ wave in solving the problem of blackout when facing different situations. The RKETD-FDTD method and model are introduced in Section 2. Results and discussions are displayed in Section 3. Conclusions are given in Section 4.

\section{RKETD-FDTD Method and Model}

For the colliding cold anisotropic magnetized plasma dispersion medium, Maxwell's equations and the associated simultaneous equations are given below

$$
\begin{gathered}
\nabla \times \mathbf{H}=\varepsilon_{0} \frac{\partial \mathbf{E}}{\partial t}+\mathbf{J} \\
\nabla \times \mathbf{E}=-\mu_{0} \frac{\partial \mathbf{H}}{\partial t} \\
\frac{d \mathbf{J}}{d t}+v \mathbf{J}=\varepsilon_{0} \omega_{p}^{2} \mathbf{E}+\omega_{b} \times \mathbf{J}
\end{gathered}
$$

where $\mathbf{J}$ refers to the polarization current density, $\varepsilon_{0}$ is the dielectric constant in vacuum, $\mu_{0}$ is the magnetic conductivity in vacuum, $v$ is the collision frequency, $\omega_{p}^{2}$ refers to the square of the angular frequency, $\omega_{b}=\mathrm{e} B_{0} / \mathrm{m}$ is the cyclotron frequency, in which $B_{0}$ is the outside magnetic field, e and $\mathrm{m}$ refer to the quantity of electric and the mass of electrons, respectively.

The expressions of $\mathbf{E}$ and $\mathbf{J}$ according to reference [23] are given directly below.

$$
\begin{gathered}
E_{x}^{n+1}\left(i+\frac{1}{2}, j, k\right)=\frac{G}{F} E_{x}^{n}\left(i+\frac{1}{2}, j, k\right)+\frac{H}{F} E_{y}^{n}\left(i+\frac{1}{2}, j, k\right)-\frac{V}{F} J_{x}^{n}\left(i+\frac{1}{2}, j, k\right)+\frac{W}{F} J_{y}^{n}\left(i+\frac{1}{2}, j, k\right) \\
+\frac{\Delta t}{\varepsilon_{0} F}\left[\frac{1}{\Delta y}\left(H_{z}^{n+\frac{1}{2}}\left(i+\frac{1}{2}, j+\frac{1}{2}, k\right)-H_{z}^{n+\frac{1}{2}}\left(i+\frac{1}{2}, j-\frac{1}{2}, k\right)\right)-\frac{1}{\Delta z}\left(H_{y}^{n+\frac{1}{2}}\left(i+\frac{1}{2}, j, k+\frac{1}{2}\right)-H_{y}^{n+\frac{1}{2}}\left(i+\frac{1}{2}, j, k-\frac{1}{2}\right)\right)\right] \\
+\frac{D \Delta t}{\varepsilon_{0} E F}\left[\frac{1}{\Delta z}\left(H_{x}^{n+\frac{1}{2}}\left(i+\frac{1}{2}, j, k+\frac{1}{2}\right)-H_{x}^{n+\frac{1}{2}}\left(i+\frac{1}{2}, j, k-\frac{1}{2}\right)\right)-\frac{1}{\Delta x}\left(H_{z}^{n+\frac{1}{2}}(i+1, j, k)-H_{z}^{n+\frac{1}{2}}(i, j, k)\right)\right]
\end{gathered}
$$




$$
\begin{aligned}
& E_{y}^{n+1}\left(i, j+\frac{1}{2}, k\right)=\frac{G}{F} E_{y}^{n}\left(i, j+\frac{1}{2}, k\right)-\frac{H}{F} E_{x}^{n}\left(i, j+\frac{1}{2}, k\right)-\frac{V}{F} J_{y}^{n}\left(i, j+\frac{1}{2}, k\right)-\frac{W}{F} J_{x}^{n}\left(i, j+\frac{1}{2}, k\right) \\
& +\frac{\Delta t}{\varepsilon_{0} F}\left[\frac{1}{\Delta x}\left(H_{z}^{n+\frac{1}{2}}\left(i, j+\frac{1}{2}, k+\frac{1}{2}\right)-H_{x}^{n+\frac{1}{2}}\left(i, j+\frac{1}{2}, k-\frac{1}{2}\right)\right)-\frac{1}{\Delta x}\left(H_{z}^{n+\frac{1}{2}}\left(i+\frac{1}{2}, j+\frac{1}{2}, k\right)-H_{z}^{n+\frac{1}{2}}\left(i-\frac{1}{2}, j+\frac{1}{2}, k\right)\right)\right] \\
& +\frac{D \Delta t}{\varepsilon_{0} E F}\left[\frac{1}{\Delta y}\left(H_{z}^{n+\frac{1}{2}}\left(i, j+\frac{1}{2}, k\right)-H_{z}^{n+\frac{1}{2}}(i, j, k)\right)-\frac{1}{\Delta z}\left(H_{y}^{n+\frac{1}{2}}\left(i, j+\frac{1}{2}, k+\frac{1}{2}\right)-H_{y}^{n+\frac{1}{2}}\left(i, j+\frac{1}{2}, k-\frac{1}{2}\right)\right)\right] \\
& E_{z}^{n+1}\left(i, j, k+\frac{1}{2}\right)=E_{z}^{n}\left(i, j, k+\frac{1}{2}\right)+\frac{\Delta t}{\varepsilon_{0}}\left[\frac{1}{\Delta x}\left(H_{y}^{n+\frac{1}{2}}\left(i+\frac{1}{2}, j, k+\frac{1}{2}\right)-H_{y}^{n+\frac{1}{2}}\left(i-\frac{1}{2}, j, k+\frac{1}{2}\right)\right)\right. \\
& \left.-\frac{1}{\Delta y}\left(H_{x}^{n+\frac{1}{2}}\left(i, j+\frac{1}{2}, k+\frac{1}{2}\right)-H_{x}^{n+\frac{1}{2}}\left(i, j-\frac{1}{2}, k+\frac{1}{2}\right)\right)-\frac{\Delta t}{2 \varepsilon_{0}}\left(J_{z}^{n+1}\left(i, j, k+\frac{1}{2}\right)+J_{z}^{n}\left(i, j, k+\frac{1}{2}\right)\right)\right] \\
& J_{x}^{n+1}\left(i, j, k+\frac{1}{2}\right)=D J_{x}^{n}\left(i+\frac{1}{2}, j, k\right)+W E_{x}^{n}\left(i+\frac{1}{2}, j, k\right)+F E_{x}^{n+1}\left(i+\frac{1}{2}, j, k\right) \\
& -G E_{y}^{n}\left(i+\frac{1}{2}, j, k\right)-H E_{y}^{n+1}\left(i+\frac{1}{2}, j, k\right)-V J_{y}^{n}\left(i+\frac{1}{2}, j, k\right) \\
& J_{y}^{n+1}\left(i, j+\frac{1}{2}, k\right)=D J_{y}^{n}\left(i+\frac{1}{2}, j, k\right)+W E_{y}^{n}\left(i+\frac{1}{2}, j, k\right)+F E_{y}^{n+1}\left(i+\frac{1}{2}, j, k\right) \\
& +G E_{x}^{n}\left(i+\frac{1}{2}, j, k\right)+H E_{x}^{n+1}\left(i+\frac{1}{2}, j, k\right)+V J_{x}^{n}\left(i+\frac{1}{2}, j, k\right) \\
& J_{z}^{n+1}\left(i, j, k+\frac{1}{2}\right)=e^{-v \Delta t} J_{z}^{n}\left(i, j, k+\frac{1}{2}\right)+\frac{\left(1-e^{-v \Delta t}\right)}{v} \varepsilon_{0} \omega_{p}^{2} E_{z}^{n}\left(i, j, k+\frac{1}{2}\right) \\
& +\frac{\left(e^{-v \Delta t}-1+v \Delta t\right)}{v^{2} \Delta t}\left[\varepsilon_{0} \omega_{\mathrm{p}}^{2} E_{z}^{n+1}\left(i, j, k+\frac{1}{2}\right)-\varepsilon_{0} \omega_{\mathrm{p}}^{2} E_{z}^{n}\left(i, j, k+\frac{1}{2}\right)\right] \\
& A=\frac{1-e^{-v \Delta t}}{v}, B=\frac{\left(e^{-v \Delta t}-1+v \Delta t\right)}{v^{2} \Delta t}, C=1+B^{2} \omega_{b}^{2} \text {, } \\
& D=\frac{\left[e^{-v \Delta t}-(A-B) B \omega_{b}^{2}\right]}{C}, W=\frac{(A-B) \varepsilon_{0} \omega_{p}^{2}}{C}, F=\frac{B \varepsilon_{0} \omega_{p}^{2}}{C}, \\
& G=\frac{B(A-B) \varepsilon_{0} \omega_{b} \omega_{p}^{2}}{C}, H=\frac{B^{2} \varepsilon_{0} \omega_{b} \omega_{p}^{2}}{C}, V=\frac{(A-B) \omega_{b}+B \omega_{b} e^{-v \Delta t}}{C} .
\end{aligned}
$$

$E_{x}, E_{y}, J_{y}$ have the same coordinates in space, $E_{y}^{n}\left(i+\frac{1}{2}, j, k\right), J_{y}^{n}\left(i+\frac{1}{2}, j, k\right) ;$ obviously, they differ from $E_{y}^{n}\left(i, j+\frac{1}{2}, k\right)$ in the standard Yee cell. So, interpolation calculations of adjacent space values are needed.

$$
\begin{gathered}
E_{y}\left(i+\frac{1}{2}, j, k\right)=\frac{1}{4}\left[E_{y}\left(i+\frac{1}{2}, j, k\right)+E_{y}\left(i, j-\frac{1}{2}, k\right)+E_{y}\left(i+1, j+\frac{1}{2}, k\right)+E_{y}\left(i+1, j-\frac{1}{2}, k\right)\right] \\
J_{y}\left(i+\frac{1}{2}, j, k\right)=\frac{1}{4}\left[J_{y}\left(i+\frac{1}{2}, j, k\right)+J_{y}\left(i, j-\frac{1}{2}, k\right)+J_{y}\left(i+1, j+\frac{1}{2}, k\right)+J_{y}\left(i+1, j-\frac{1}{2}, k\right)\right]
\end{gathered}
$$

For the magnetic field, the average amounts of the four surrounding points are calculated, respectively,

$$
\begin{aligned}
H_{x}^{n+\frac{1}{2}}(i+ & \left.\frac{1}{2}, j, k+\frac{1}{2}\right)=\frac{1}{4}\left[H_{x}^{n+\frac{1}{2}}\left(i, j-\frac{1}{2}, k+\frac{1}{2}\right)+H_{x}^{n+\frac{1}{2}}\left(i, j+\frac{1}{2}, k+\frac{1}{2}\right)\right. \\
& \left.+H_{x}^{n+\frac{1}{2}}\left(i+, j-\frac{1}{2}, k+\frac{1}{2}\right)+H_{x}^{n+\frac{1}{2}}\left(i+1, j+\frac{1}{2}, k+\frac{1}{2}\right)\right] \\
H_{x}^{n+\frac{1}{2}}\left(i+\frac{1}{2}, j, k-\frac{1}{2}\right)= & \frac{1}{4}\left[H_{x}^{n+\frac{1}{2}}\left(i, j-\frac{1}{2}, k-\frac{1}{2}\right)+H_{x}^{n+\frac{1}{2}}\left(i, j+\frac{1}{2}, k-\frac{1}{2}\right)\right. \\
+ & \left.H_{x}^{n+\frac{1}{2}}\left(i+1, j-\frac{1}{2}, k-\frac{1}{2}\right)+H_{x}^{n+\frac{1}{2}}\left(i+1, j+\frac{1}{2}, k-\frac{1}{2}\right)\right]
\end{aligned}
$$




$$
\begin{gathered}
H_{z}^{n+\frac{1}{2}}(i+1, j, k)=\frac{1}{4}\left[H_{z}^{n+\frac{1}{2}}\left(i+\frac{1}{2}, j-\frac{1}{2}, k\right)+H_{z}^{n+\frac{1}{2}}\left(i+\frac{1}{2}, j+\frac{1}{2}, k\right)\right. \\
\left.+H_{z}^{n+\frac{1}{2}}\left(i+\frac{3}{2}, j-\frac{1}{2}, k\right)+H_{z}^{n+\frac{1}{2}}\left(i+\frac{3}{2}, j+\frac{1}{2}, k\right)\right] \\
H_{z}^{n+\frac{1}{2}}(i, j, k)=\frac{1}{4}\left[H_{z}^{n+\frac{1}{2}}\left(i-\frac{1}{2}, j-\frac{1}{2}, k\right)+H_{z}^{n+\frac{1}{2}}\left(i-\frac{1}{2}, j+\frac{1}{2}, k\right)\right. \\
\left.+H_{z}^{n+\frac{1}{2}}\left(i+\frac{1}{2}, j-\frac{1}{2}, k\right)+H_{z}^{n+\frac{1}{2}}\left(i+\frac{1}{2}, j+\frac{1}{2}, k\right)\right]
\end{gathered}
$$

$E_{x}, E_{y}, J_{y}$ have the same coordinates in space, $E_{x}^{n}\left(i, j+\frac{1}{2}, k\right), J_{x}^{n}\left(i, j+\frac{1}{2}, k\right)$; obviously, they differ from $E_{x}^{n}\left(i+\frac{1}{2}, j, k\right)$ in the standard Yee cell. So, interpolation calculations of adjacent space values are needed.

$$
\begin{aligned}
& E_{x}^{n}\left(i, j+\frac{1}{2}, k\right)=\frac{1}{4}\left[E_{x}^{n}\left(i+\frac{1}{2}, j, k\right)+E_{x}^{n}\left(i, j-\frac{1}{2}, k\right)+E_{x}^{n}\left(i+\frac{1}{2}, j+1, k\right)+E_{x}^{n}\left(i-\frac{1}{2}, j+1, k\right)\right] \\
& J_{x}^{n}\left(i, j+\frac{1}{2}, k\right)=\frac{1}{4}\left[J_{x}^{n}\left(i-\frac{1}{2}, j, k\right)+J_{x}^{n}\left(i-\frac{1}{2}, j+1, k\right)+J_{x}^{n}\left(i+\frac{1}{2}, j, k\right)+J_{x}^{n}\left(i+\frac{1}{2}, j+1, k\right)\right] \\
& H_{z}^{n+\frac{1}{2}}(i+1, j+1, k)=\frac{1}{4}\left[H_{z}^{n+\frac{1}{2}}\left(i-1, j+\frac{1}{2}, k\right)+H_{z}^{n+\frac{1}{2}}\left(i-\frac{1}{2}, j+\frac{3}{2}, k\right)\right. \\
& \left.+H_{z}^{n+\frac{1}{2}}\left(i+\frac{1}{2}, j+\frac{1}{2}, k\right)+H_{z}^{n+\frac{1}{2}}\left(i+\frac{1}{2}, j+\frac{3}{2}, k\right)\right] \\
& H_{z}^{n+\frac{1}{2}}(i, j, k)=\frac{1}{4}\left[H_{z}^{n+\frac{1}{2}}\left(i-\frac{1}{2}, j-\frac{1}{2}, k\right)+H_{z}^{n+\frac{1}{2}}\left(i-\frac{1}{2}, j+\frac{1}{2}, k\right)\right. \\
& \left.+H_{z}^{n+\frac{1}{2}}\left(i+\frac{1}{2}, j-\frac{1}{2}, k\right)+H_{z}^{n+\frac{1}{2}}\left(i+\frac{1}{2}, j+\frac{1}{2}, k\right)\right] \\
& H_{y}^{n+\frac{1}{2}}\left(i, j+\frac{1}{2}, k+\frac{1}{2}\right)=\frac{1}{4}\left[H_{y}^{n+\frac{1}{2}}\left(i-\frac{1}{2}, j, k+\frac{1}{2}\right)+H_{z}^{n+\frac{1}{2}}\left(i-\frac{1}{2}, j+1, k+\frac{1}{2}\right)\right. \\
& \left.+H_{y}^{n+\frac{1}{2}}\left(i+\frac{1}{2}, j, k+\frac{1}{2}\right)+H_{y}^{n+\frac{1}{2}}\left(i+\frac{1}{2}, j+1, k+\frac{1}{2}\right)\right] \\
& H_{y}^{n+\frac{1}{2}}\left(i, j+\frac{1}{2}, k-\frac{1}{2}\right)=\frac{1}{4}\left[H_{y}^{n+\frac{1}{2}}\left(i-\frac{1}{2}, j, k-\frac{1}{2}\right)+H_{z}^{n+\frac{1}{2}}\left(i-\frac{1}{2}, j+1, k-\frac{1}{2}\right)\right. \\
& \left.+H_{y}^{n+\frac{1}{2}}\left(i+\frac{1}{2}, j, k-\frac{1}{2}\right)+H_{y}^{n+\frac{1}{2}}\left(i+\frac{1}{2}, j+1, k-\frac{1}{2}\right)\right]
\end{aligned}
$$

Similarly, if we want to get $J_{x}^{n+1}\left(i+\frac{1}{2}, j, k\right)$ from Equation (7), $E_{y}^{n+1}\left(i+\frac{1}{2}, j, k\right)$, $E_{y}^{n}\left(i+\frac{1}{2}, j, k\right)$ and $J_{y}^{n}\left(i+\frac{1}{2}, j, k\right)$ are needed and they are not in the node positions of the corresponding component in the Yee cell. Applying spatial interpolation according to the node position of the corresponding component in the Yee cell is necessary. For Equation (8), $J_{y}^{n+1}\left(i, j+\frac{1}{2}, k\right)$ is calculated by $E_{x}^{n+1}\left(i, j+\frac{1}{2}, k\right), E_{x}^{n}\left(i+\frac{1}{2}, j, k\right)$ and $J_{x}^{n}\left(i, j+\frac{1}{2}, k\right)$. It has the same solution process as $J_{x}^{n+1}\left(i+\frac{1}{2}, j, k\right)$.

Due to limited computer resources, RCS is calculated in a limited field. The side length of the total FDTD calculation field is 80 cells, and the perfectly matched layer (PML) absorbing boundary is set to 5 cells [25]. The time step in simulation $d t=9.8 \times 10^{-14} \mathrm{~s}$ and the space step cell $\delta=5.9 \times 10^{-5} \mathrm{~m}$. Relative electrically small models are used to compute the RCS [26]. Simulations in most of the study are based on the 3D von Karman conductive model. Figure 1 is the schematic of the model. 
(a)

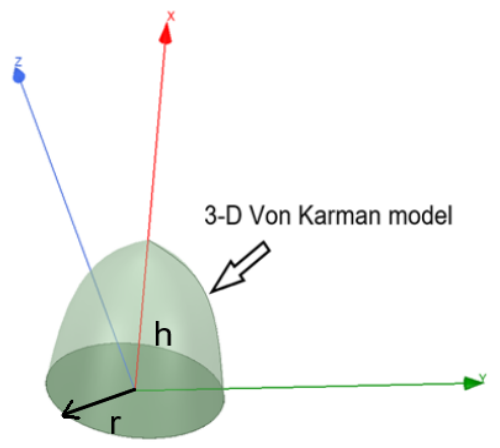

(b)

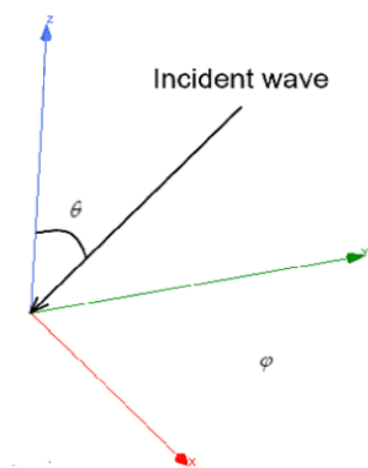

Figure 1. (a) Finite difference time domain (FDTD) for computing the radar cross section (RCS) of the von Karman model; (b) Direction of the incident wave and scan angle.

Symbol $\mathrm{h}$ refers to the height of the model, which is 20 cells, and $\mathrm{r}$ is set as the radius of the circle at the bottom with a length of 10 cells. Plasma is applied to the surface of the model with a thickness of $d . \theta$ is the angle between incident wave and the positive semi-axis of the $z$ coordinate and $\varphi$ is for RCS scanning from 0 to 360 degree in the $x-y$ plane.

Using the FDTD method to calculate the RCS follows the steps below: Derivation of iterative equation of scattering field, setting incident plane wave source, absorbing boundary, near-field to far-field outside derivation and calculation of the conductive model. The RCS defining formula is

$$
\sigma=\lim _{R \rightarrow \infty} 4 \pi R^{2} \frac{\left|E_{s}\right|^{2}}{\left|E_{i}\right|^{2}}
$$

$E_{s}$ represents the scattering electric field and $E_{i}$ is the incident electric field; $R$ is the distance between the radar and the target.

\section{Results and Discussions}

\subsection{Attenuation and Monostatic RCS}

In this part, the monostatic RCS is discussed to illustrate the increasing RCS function of the EM wave at $0.34 \mathrm{THz}$. As the radio wave travels through the plasma, free electrons and ions vibrate back and forward; their energy comes from the EM wave. Vibrating electrons and ions collide with neutral particle, thus causing the energy loss of EM waves. Before RCS calculation, firstly, the propagation mechanism of the electromagnetic wave in plasma is introduced [27]. Considering the parameters of dense plasma formed in the re-entry process introduced in relative works [1,28], the electron density of the homogeneous plasma is set as $1 \times 10^{19} \mathrm{~m}^{-3}$ and the collisional frequency is $0.1 \mathrm{THz}$ in the simulation; the magnetic field is applied in the $Z$ direction with the cyclotron resonance $2 \times 10^{11} \mathrm{rad} / \mathrm{s}, \theta$ is 90 degrees and the incident wave is applied along the negative $x$ semi-axis. The relationship of plasma frequency and density $N_{\mathrm{e}}$ are given below [29].

$$
\omega_{\mathrm{p}} \approx \omega_{\mathrm{pe}}=\sqrt{\frac{N_{e} e^{2}}{\varepsilon_{0} m_{e}}}
$$

$\varepsilon_{0}$ is the dielectric constant in vacuum, $m_{e}$ is the mass of the electron and $e$ represents the electric charge of the electron. The attenuation constant $\alpha$ [30]

$$
\alpha=\frac{\omega}{\mathrm{c}} \frac{1}{\sqrt{2}}\left\{\left(\frac{\omega_{p}^{2}}{v^{2}+\omega^{2}}-1\right)+\left[\left(1-\frac{\omega_{p}^{2}}{v^{2}+\omega^{2}}\right)^{2}+\frac{\omega_{p}^{2} v}{v^{2}+\omega^{2}}\right]^{\frac{1}{2}}\right\}^{\frac{1}{2}}
$$

$\omega$ is the angular frequency, $c$ is the light speed and $v$ is the collisional frequency in expression (24). Using the attenuation constant expression, the return attenuation is 
further discussed with the WKB method. For the incident wave that enters the homogeneous plasma at $z=0$, spreading to a reflecting target at $z=z_{0}$, the WKB approximation concerning the return attenuation in $\mathrm{dB}$ is

$$
A T T=\left|10 \lg \frac{P\left(Z_{0}\right)}{P_{0}}\right|=\left|17.37\left(\int_{0}^{z_{0}} k(z) d z\right)\right|
$$

where the wave number $k$ is given by the expression

$$
k=k_{0} \sqrt{\varepsilon_{r}}=\beta-j \alpha
$$

$k_{0}$ is the wave number in free space, $p_{0}$ is the power at $z=0, \alpha$ is the attenuation constant, $\beta$ is the phase constant. The attenuation results are shown in Figure 2.

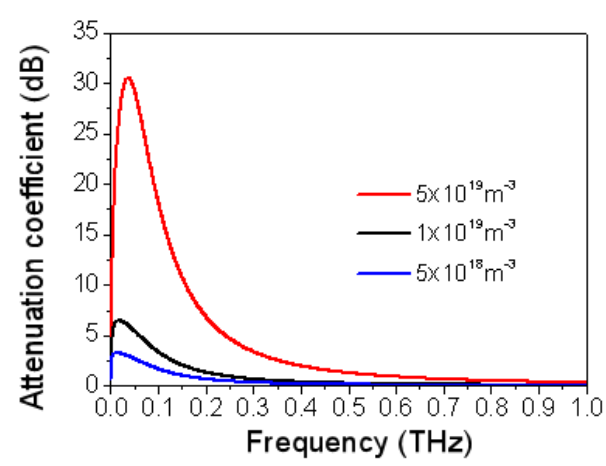

Figure 2. The attenuation coefficient varies with incident wave frequency and plasma density.

The results in Figure 2 correspond to the analysis that a larger density of plasma has a higher attenuation. While the frequency of the incident wave is raised from 0.05 to $1 \mathrm{THz}$, the attenuation decreases. However, the peaks of the curves of different plasma densities move to higher incident frequency in the range of $0-0.05 \mathrm{THz}$. It can be explained that plasma with higher density has a higher cut-off frequency, which makes the EM wave below the cut-off frequency reflect straight and have less attenuation. As can be seen from Figure 3, due to the high-pass characteristics of plasma, RCS is not increased by an EM wave of $0-0.05 \mathrm{THz}$. In the range of $0.05-0.3 \mathrm{THz}$, RCS is obviously reduced by the magnetized plasma, while two curves are close when the frequency is greater than $0.3 \mathrm{THz}$. Therefore, for the frequency of $0.34 \mathrm{THz}$, the EM wave absolutely has less attenuation than a microwave and a low- $\mathrm{THz}$ wave.

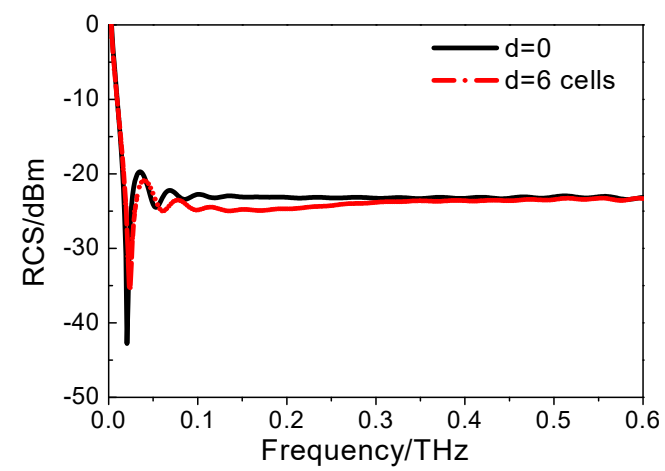

Figure 3. Monostatic RCS of the model with different thicknesses of plasma.

\subsection{Electric Field Polarization and Thickness of Plasma}

In this section, the frequency of the incident wave is fixed at $0.34 \mathrm{THz}$ and the bistatic RCS in different polarization ways of horizontal and vertical polarizations is calculated. Additionally, both magnetized and unmagnetized plasma are discussed. The electron 
density of the homogeneous plasma is set as $1 \times 10^{19} \mathrm{~m}^{-3}$ and the collisional frequency is $0.1 \mathrm{THz}$ in the simulation. The magnetic field is applied in the $z$ direction with the cyclotron resonance $2 \times 10^{11} \mathrm{rad} / \mathrm{s}, \theta$ is 90 degree and the incident wave is applied along the $x$ negative directions. Results are displayed in Figure 4 .
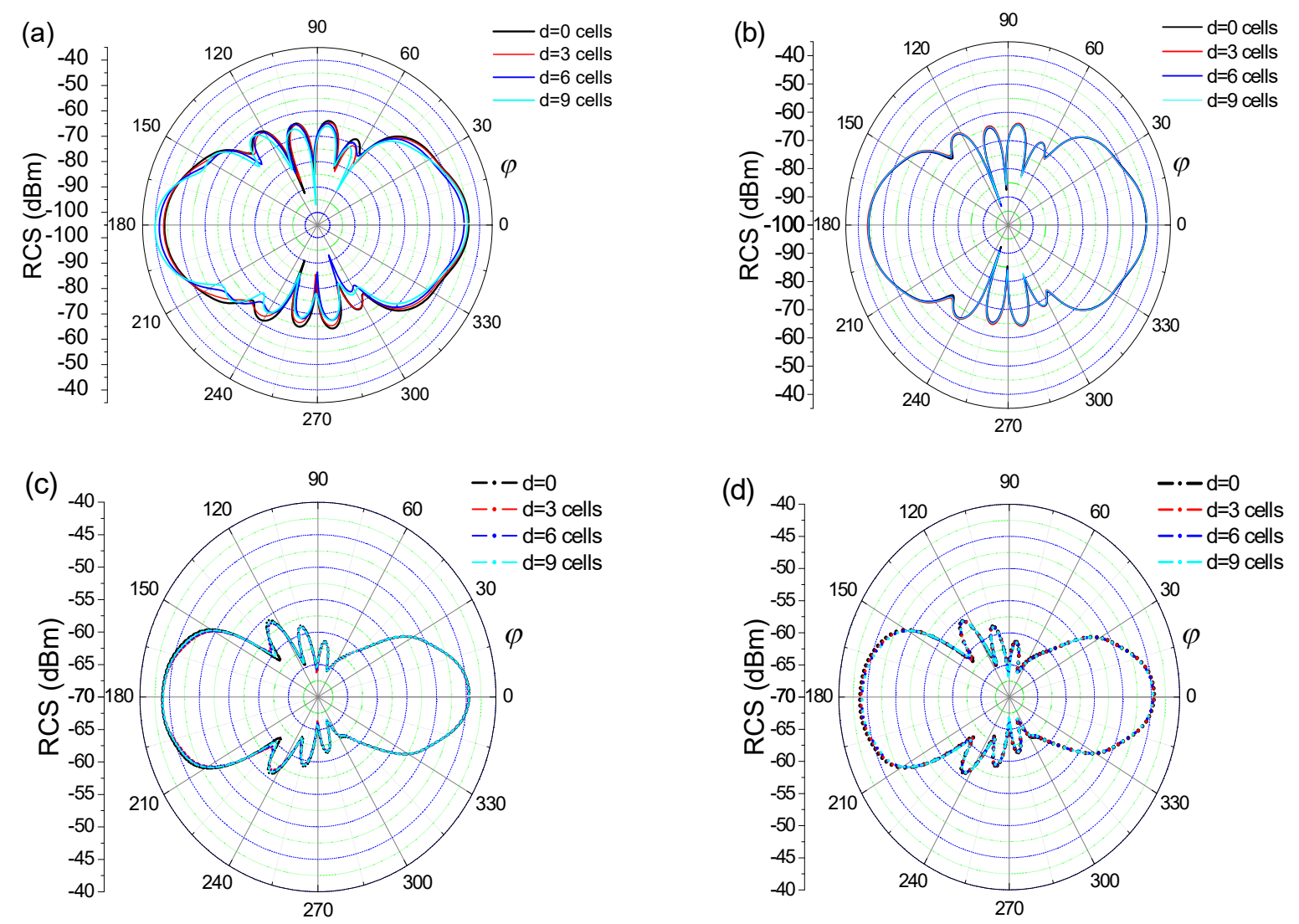

Figure 4. (a) Bistatic RCS in polar coordinates with magnetized plasma and horizontal polarization. (b) RCS with unmagnetized plasma and horizontal polarization. (c) RCS with magnetized plasma and vertical polarization. (d) RCS with unmagnetized plasma and vertical polarization.

In Figure $4 a$, with the increase of the thickness of plasma d, the RCS becomes lower, which means the EM wave has more attenuation in the thicker plasma. The RCS at 180 bistatic degrees is slightly greater than the RCS calculated without plasma. As for the von Karman model established, the bistatic RCS near the bistatic angle 180 degrees can be regarded proportional to the target projection area. If we take the conductor cylinder and magnetized plasma into consideration, the projection area truly increases. Additionally, the magnetic field is applied in the $z$ direction. The electric field couples and plasma is anisotropy; therefore, the bistatic RCS is asymmetrical in $\varphi$ from 0 to 360 degrees. Combining Figure $4 a, b$, it can be found that the RCS of unmagnetized plasma is higher than the RCS of magnetized plasma when simulated in horizontal polarization. As shown in Figure $4 \mathrm{c}, \mathrm{d}$, in vertical polarization, no matter whether the plasma is magnetized or not, the RCSs of different thicknesses of plasma have almost the same values as the results calculated with $d=0$. This should be explained from the polarization mechanism: The longitudinal component $\mathrm{E}_{\mathrm{x}}$ and the transverse component $\mathrm{E}_{\mathrm{y}}$ of horizontal polarization combine as the hybrid wave and the plasma is anisotropy. However, the plasma in vertical polarization shows isotropy. For plasma magnetization and electric field polarization, some of the contents in this part are consistent with those in the literature [31].

Generally speaking, the bistatic RCS of $0.34 \mathrm{THz}$ simulated with different plasma and polarizations are close to the curve with $d=0$, which is corresponding to the monostatic 
RCS results. $0.34 \mathrm{THz}$ has polarization insensitivity and strong penetration ability when the thickness $d$ increases in a certain range.

\subsection{Incident Directions}

The incident direction of $0.34 \mathrm{THz}$ wave is discussed in this section. In this section, the electron density of the homogeneous plasma is set as $1 \times 10^{19} \mathrm{~m}^{-3}$ and the collisional frequency is $0.1 \mathrm{THz}$ in the simulation, the magnetic field is applied to $\mathrm{Z}$ direction with the cyclotron resonance $2 \times 10^{11} \mathrm{rad} / \mathrm{s}, d=6$ cells and the electrical field is vertical polarization. Results are shown in Figures 5 and 6.
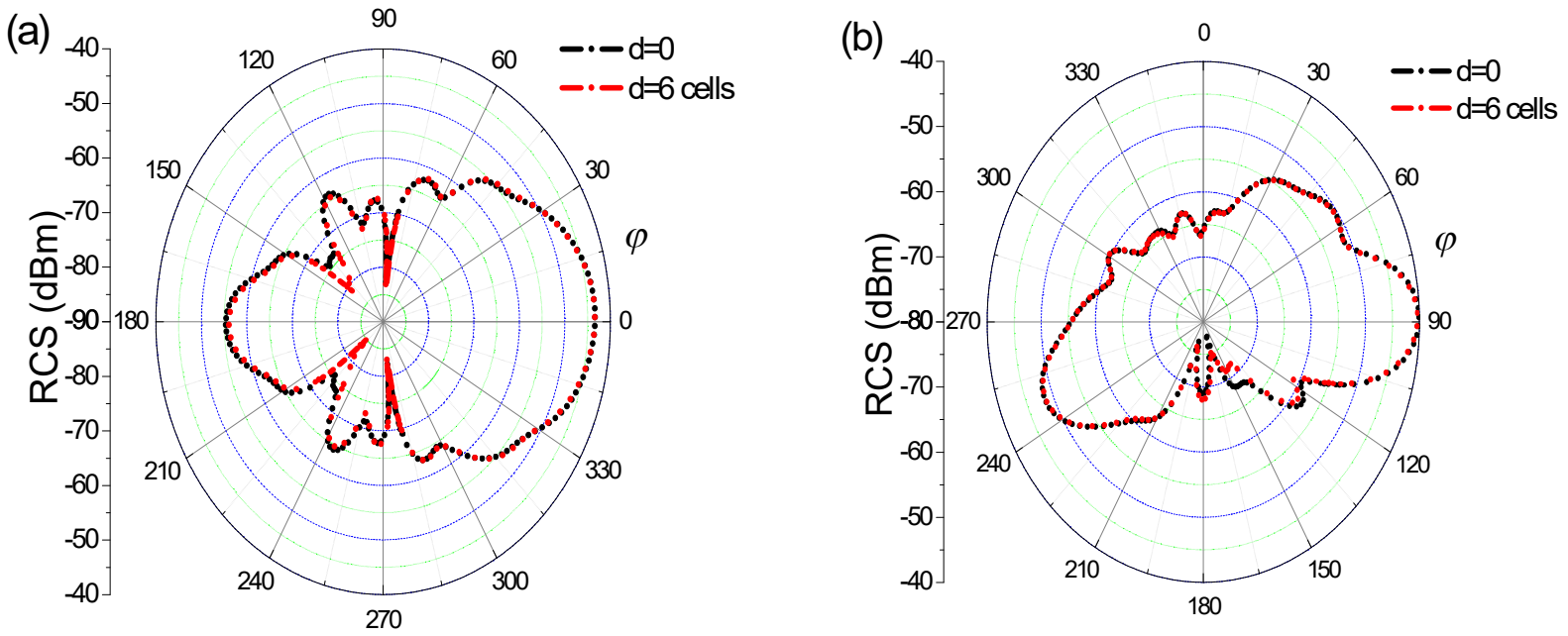

Figure 5. (a) Bistatic RCS with the incident wave along the $x$ positive axis. (b) Bistatic RCS with different thicknesses of plasma when the incident wave is along the $y$ positive axis.
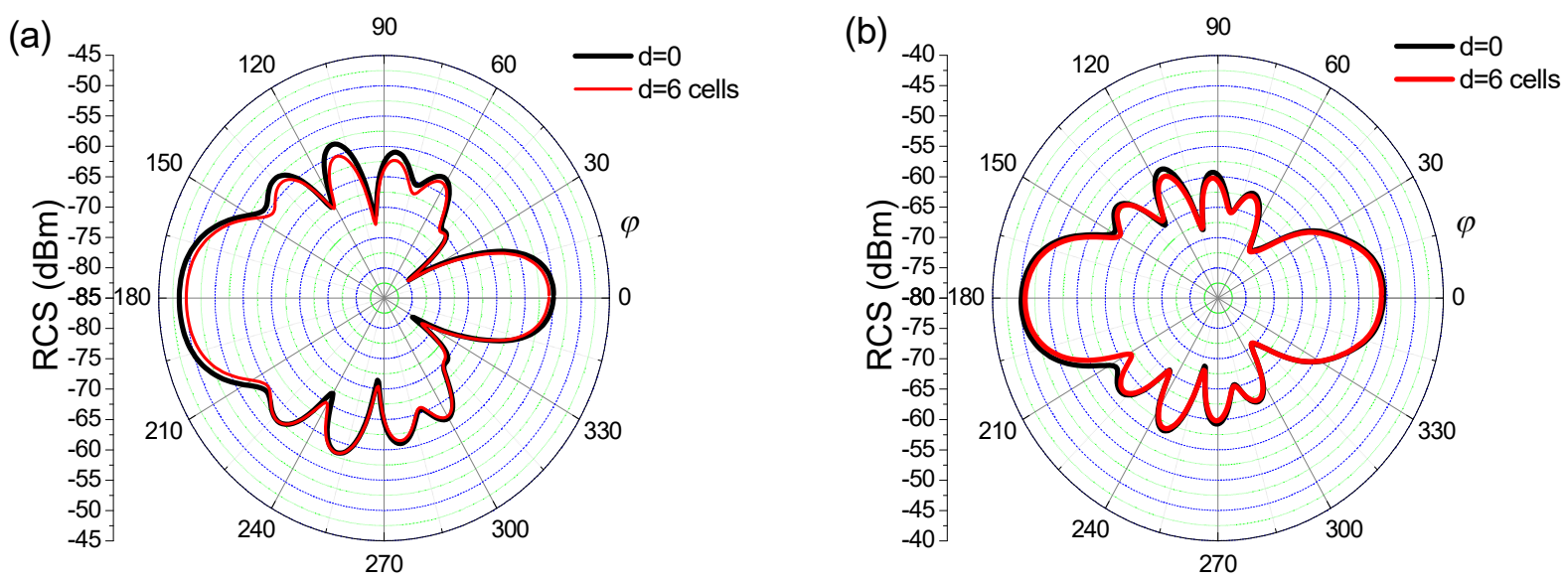

Figure 6. (a) Bistatic RCS with $\theta=45$ degrees (b) Bistatic RCS with $\theta=60$ degrees.

It is obvious that the RCS will be changed when the incident angle is changed. We discussed the influence of the incident directions of $0.34 \mathrm{THz}$ wave. RCS is scanned in $x-y$ plane for $\varphi$ from 0 to 360 degree. From simulation results abovementioned in Figure 5, it can be concluded that although the curve shapes are changing dramatically, bistatic RCS of $0.34 \mathrm{THz}$ has almost the same value compared with the $d=0$ situation.

The bistatic RCS is discussed with the change of $\theta$ in Figure 6. The results show that the bistatic RCS reduces slightly when the model is coated with the magnetized plasma. For EM waves coming in from different directions, $0.34 \mathrm{THz}$ can have good performances in these different simulation situations. When the re-entry vehicles are moving in the near space, the directions will change quickly and $0.34 \mathrm{THz}$ may have a better penetration in the whole re-entry progress. 


\subsection{Parabolic Time-Varying Plasma}

In fact, the distribution of free electron may not be always uniform. In the low ionosphere, distribution of free electron density can be simulated by parabolic and time-varying distribution [32]. Formulas below are the angular frequency expressions of parabolic and time-varying distribution plasma:

$$
\omega_{\mathrm{p}}(l, t)=\left(\omega_{\mathrm{p} 1}+\omega_{\mathrm{p} 2} \frac{l}{d}\right) \frac{t}{T_{r}}
$$

In expressions (27), $l$ represents the vertical distance between any point in plasma and the surface of the conductive target, $t$ is the time step, $d$ is the thickness of the plasma, $T_{r}$ is the relaxation time and $\omega_{p 1}=2 \pi \times 10^{10} \mathrm{rad} / \mathrm{s}, \omega_{p 2}=20 \pi \times 10^{10} \mathrm{rad} / \mathrm{s}$, respectively. For simulation conditions, the collisional frequency is $0.1 \mathrm{THz}$ in the simulation, the magnetic field is applied to $Z$ direction with the cyclotron resonance $2 \times 10^{11} \mathrm{rad} / \mathrm{s}, \theta$ is 90 degree, and the incident wave is applied along $x$ negative semi-axis. The RCS simulated parabolic plasma and its relaxation time are displayed in Figure 7.

(a)

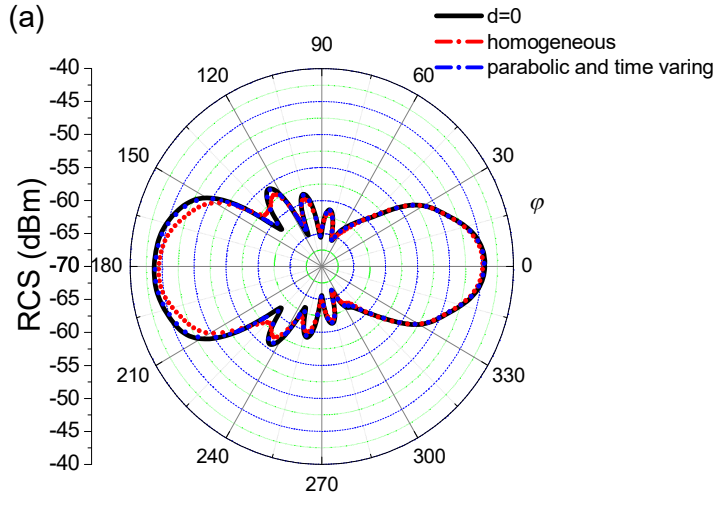

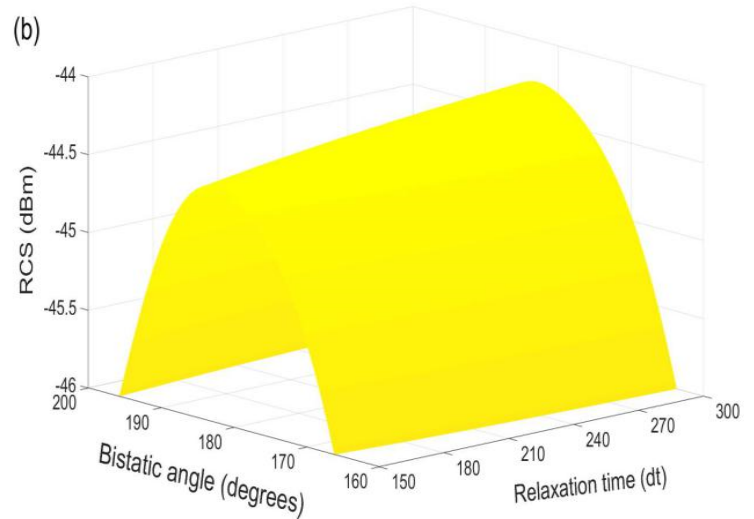

Figure 7. (a) RCS with different distributions of plasma in magnetized plasma (relaxation time $150 \mathrm{dt}$ ). (b) RCS versus relaxation time (160-200 bistatic degrees).

In Figure 7a, RCS with homogeneous plasma is close to the curve $d=0$, while the parabolic and time-varying plasma curve has a lower RCS in the bistatic angle from 150 to 210 degrees. Inhomogeneous plasma can reduce the RCS more significantly than homogeneous plasma. This is because inhomogeneous plasma is a gradual changing and discontinuous distribution and the electromagnetic wave is refracted, absorbed and weakened after entering the plasma. The targets have fewer echoes and smaller RCS. However, $0.34 \mathrm{THz}$ can increase the RCS in both homogeneous and parabolic and timevarying distribution plasmas because they have almost the same values in most bistatic angles $\varphi$ from 0 to 360 degrees. In Figure $7 \mathrm{~b}$, the influence of the relaxation time is discussed and displayed. As the relaxation time increases from 150 to $300 \mathrm{dt}$, there is a slight strengthening in bistatic RCS near 180 bistatic degrees, which means larger relaxation time has less attenuation. For this phenomenon, lager relaxation time makes plasma take more time to reach the max density and reduce the absorption of EM waves. Concerning the results and phenomena shown in Figure 6, they are consistent with the research work in reference [17].

\section{Conclusions}

In this paper, increasing the frequency of the incident wave to solve the blackout problem and realizability concerning $0.34 \mathrm{THz}$ in telecommunications are introduced. The derivation of the numerical method is introduced in detail. The interaction between the $\mathrm{THz}$ wave and magnetized and inhomogeneous plasma is discussed based on the method. Attenuation of EM wave propagating in plasma is discussed using the WKB method 
and used as a theoretical base. Using the RKETD-FDTD numerical method extended to three dimensions, monostatic RCS is calculated to illustrate that raising the frequency to $0.34 \mathrm{THz}$ can reduce the attenuation more than microwave and low- $\mathrm{THz}$ (around $150 \mathrm{GHz}$ ) and bistatic RCS of $0.34 \mathrm{THz}$ to discuss its advantages for enabling telecommunications. We establish a von Karman model to calculate the RCS in different simulation conditions. Results of the bistatic RCS of $0.34 \mathrm{THz}$ exhibit excellent performance in different polarizations of the electric field, parabolic and time-varying plasma sheath, different thicknesses of the plasma, the magnetic field applied situations, different incident angles and different planes for RCS calculation. Additionally, physical explanations are made and comparisons with previous work for the phenomena are shown in figures displayed to verify the accuracy of the simulation; $0.34 \mathrm{THz}$ can make the numerical results of RCS almost the same as results simulated when the thickness of the plasma is 0 . Generally speaking, increasing frequency is actually a realizable and effective way to solve the blackout problem. Terahertz waves at $0.34 \mathrm{THz}$ are effective tools for keeping telecommunication unobstructed when faced with complex situations.

Author Contributions: Conceptualization, H.L.; methodology, H.L. and P.W.; software, H.L. and P.W.; writing — original draft preparation, H.L.; writing—review and editing, J.W. and X.Y.; supervision, Y.Z. and X.Z.; project administration, Y.Z. and X.Z. All authors have read and agreed to the published version of the manuscript.

Funding: This work was supported by the Research Innovation Fund for College Students of Beijing University of Posts and Telecommunications, and the Fund of State Key Laboratory of Information Photonics and Optical Communications (Beijing University of Posts and Telecommunications), P. R. China (IPOC2020ZZ01 and IPOC2019ZT07).

Institutional Review Board Statement: Not applicable.

Informed Consent Statement: Not applicable.

Data Availability Statement: All simulated data except for the $\mathrm{C}++$ code is available to the readers.

Conflicts of Interest: The authors declare no conflict of interest.

\section{References}

1. Yuan, K.; Shen, L.; Yao, M.; Deng, X.; Chen, Z.; Hong, L. Studies on the transmission of sub-THz waves in magnetized inhomogeneous plasma sheath. Phys. Plasmas 2018, 25, 013302. [CrossRef]

2. Rybak, J.; Churchill, R.J. Progress in reentry communications. Aerosp. Electron. Syst. IEEE Trans. 1971, AES-7, 879-894.

3. Keidar, M.; Kirn, M.; Boyd, I.D. Electromagnetic reduction of plasma density during atmospheric reentry and hypersonic flights. J. Spacecr. Rocket. 2008, 45, 445-453. [CrossRef]

4. Kim, M.; Keidar, M.; Boyd, I.D. Analysis of an electromagnetic mitigation scheme for reentry telemetry through plasma. J. Spacecr. Rocket. 2008, 45, 1223-1229. [CrossRef]

5. Kundrapu, M.; Loverich, J.; Beckwith, K.; Stoltz, P.; Keidar, M. Electromagnetic wave propagation in the plasma layer of a reentry vehicle. In Proceedings of the IEEE 41st International Conference on Plasma Sciences (ICOPS) held with 2014 IEEE International Conference on High-Power Particle Beams (BEAMS), Washington, DC, USA, 25-29 May 2014; pp. 1-4.

6. Kundrapu, M.; Loverich, J.; Beckwith, K.; Stoltz, P.; Shashurin, A.; Keidar, M. Modeling radio communication blackout and blackout mitigation in hypersonic vehicles. J. Spacecr. Rocket. 2015, 52, 853-862. [CrossRef]

7. Hartunian, R.; Stewart, G.; Curtiss, T.; Fergason, S.; Seibold, R.; Shome, P. Implications and Mitigation of Radio Frequency Blackout During Reentry of Reusable Launch Vehicles. In Proceedings of the AIAA Atmospheric Flight Mechanics Conference and Exhibit, Hilton Head, SC, USA, 20-27 August 2007.

8. Zheng, L.; Zhao, Q.; Liu, S.; Xing, X.; Chen, Y. Theoretical and experimental studies of terahertz wave propagation in unmagnetized plasma. J. Infrared Millim. Terahertz Waves 2014, 35, 187-197. [CrossRef]

9. Schexnayder, C.J.; Evans, J.S.; Huber, P.W. Comparison of Theoretical and Experimental Electron Density for RAM C Flights. NASA Spec. Publ. 1971, 252, 277.

10. Wang, C.; Lu, B.; Lin, C.; Chen, Q. 0.34-THz Wireless Link Based on High-Order Modulation for Future Wireless Local Area Network Applications. IEEE Trans. Terahertz Sci. Technol. 2014, 4, 75-85. [CrossRef]

11. Liu, Y.; Zhang, B.; Feng, Y.; Lv, X.; Ji, D.; Niu, Z.; Yang, Y.; Zhao, X.; Fan, Y. Development of 340-GHz Transceiver Front End Based on GaAs Monolithic Integration Technology for THz Active Imaging Array. Appl. Sci. 2020, 10, 7924. [CrossRef]

12. Wang, M.; Yu, M.; Xu, Z.; Li, G.; Jiang, B.; Xu, J. Propagation properties of terahertz waves in a time-varying dusty plasma slab using fdtd. IEEE Trans. Plasma Sci. 2015, 43, 4182-4186. [CrossRef] 
13. Yuan, C.X.; Zhou, Z.X.; Zhang, J.W.; Xiang, X.L.; Sun, H.G. Fdtd analysis of terahertz wave propagation in a high-temperature unmagnetized plasma slab. IEEE Trans. Plasma Sci. 2011, 39, 1577-1584. [CrossRef]

14. Kang, Z.; Huang, M.; Li, W.; Wang, Y.; Yang, F. An Efficient Numerical Formulation for Wave Propagation in Magnetized Plasma Using PITD Method. Electronics 2020, 9, 1575. [CrossRef]

15. Knott, E.F.; Schaeffer, J.F.; Tulley, M.T. Radar Cross Section; SciTech Publishing: Raleigh, NC, USA, 2004; pp. 1-3.

16. Huang, H.R.; Wang, H.W.; Sun, P.; Cui, Y. Research on Electromagnetic Scattering Characteristics and Radar Cross Section Reduction of Vertical tail. In Proceedings of the 2015 6th International Conference on Manufacturing Science and Engineering, Guangzhou, China, 28-29 November 2015.

17. Liu, S.B.; Liu, S.Q.; Yuan, N.C. Fdtd simulation of bistatic scattering by conductive cylinder covered with inhomogeneous time-varying plasma. Plasma Sci. Technol. 2006, 8, 190-194.

18. Liu, S.; Wu, P.; Zhong, S.Y.; Zhao, S.Y. Numerical calculation of the RCS of conductive objects covered with time-varying inhomogeneous plasma. In Proceedings of the IEEE International Conference on Microwave Technology \& Computational Electromagnetics, Beijing, China, 22-25 May 2011.

19. Liu, S.; Zhong, S.Y. Analysis of backscattering RCS of targets coated with parabolic distribution and time-varying plasma media. Optik Int. J. Light Electron Opt. 2013, 124, 6850-6852. [CrossRef]

20. Sanchez-Olivares, P.; Lozano, L.; Somolinos, Á.; Cátedra, F. EM Modelling of Monostatic RCS for Different Complex Targets in the Near-Field Range: Experimental Evaluation for Traffic Applications. Electronics 2020, 9, 1890. [CrossRef]

21. Dai, H.; Zhao, Y.; Li, H.; Chen, J.; He, Z.; Qi, W. An Ultra-Wide Band Polarization-Independent Random Coding Metasurface for RCS Reduction. Electronics 2019, 8, 1104. [CrossRef]

22. Liu, S.; Zhong, S.Y. FDTD Study on Scattering for Conducting Target Coated with Magnetized Plasma of Time-Varying Parabolic Density Distribution. Prog. Electromagn. Res. M 2012, 22, 13-25. [CrossRef]

23. Liu, S.; Zhong, S.Y.; Liu, S.B. Finite-difference time-domain algorithm for dispersive media based on Runge-Kutta exponential time differencing method. Int. J. Infrared Millim. Waves 2008, 29, 323-328. [CrossRef]

24. Cox, S.M.; Matthews, P.C. Exponential time differencing for stiff systems. J. Comput. Phys. 2002, 176, 430-455. [CrossRef]

25. Liu, S.B.; Zhou, T.; Li, M.L.; Hong, W. Wentzel-Kramer-Brillouin and finite-difference time-domain analysis of Terahertz band electromagnetic characteristics of target coated with unmagnetized plasma. J. Syst. Eng. Electron. 2008, 19, 15-20.

26. Hastings, F.D.; Schneider, J.B.; Broschat, S.L. Application of the perfectly matched layer (PML) absorbing boundary condition to elastic wave propagation. J. Acoust. Soc. Am. 1996, 100, 3061-3069. [CrossRef]

27. Lin, T.C.; Sproul, L.K. Influence of reentry turbulent plasma fluctuation on EM wave propagation. Comput. Fluids 2006, 35, 703-711. [CrossRef]

28. Yuan, K.J.; Chen, L.; Shen, X.; Deng, Y.; Ming, L. Hong Impact of Reentry of reentry speed on the transmission of obliquely incident THz waves in realistic plasma sheaths. IEEE Trans. Plasma Sci. 2018, 46, 373-378. [CrossRef]

29. Zhong, Y.M.; Chen, M.; Li, M.H.; Li, Y.H. Attenuation model and simulation validation of electromagnetic wave transmission in reentry plasma. J. Telem. Track. Command 2010, 31, 3-8.

30. Russo, A.J. Estimates of Attenuation and Reflection of Telemetering Signals by Ionized Flow Fields Surrounding Typical Reentry Bodies; NASA-TN-D-1778; National Aeronautics and Space Administration: Washington, DC, USA, 1963.

31. Xu, L.J.; Yuan, N.C. FDTD Formulations for Scattering From 3-D Anisotropic Magnetized Plasma Objects. IEEE Antennas Wirel. Commun. Propag. Lett. 2006, 5, 335-338. [CrossRef]

32. Liu, S.; Mo, J.; Yuan, N. FDTD Simulation of Electromagnetic Reflection of Conductive Plane Covered with Inhomogeneous Time-Varying Plasma. Int. J. Infrared Millim. Waves 2002, 23, 1179-1191. [CrossRef] 